\title{
Time duration to safety sitting in parturient receiving spinal anesthesia for cesarean section with $0.5 \%$ Bupivacaine and morphine
}

\author{
Sahatsa Mandee, Arunotai Siriussawakul, Suwannee Suraseranivongse, Jongrak Khanwilai, Pongsak Nitigarun \\ Department of Anesthesiology, Faculty of Medicine, Siriraj Hospital, Mahidol University, Bangkok \\ 10700, Thailand
}

\begin{abstract}
Background: Spinal anesthesia has been used for cesarean section for a long time. However, the proportion of post-cesarean paturients who were able to sit at the fourth hour still remains unclear.

Objective: Investigate the proportion of post-cesarean paturients that were able to sit at the fourth hour following spinal anesthesia with $0.5 \%$ hyperbaric bupivacaine and morphine. Furthermore, investigate the optimum time to encourage ambulation, and the risk factors delaying time duration to sit.

Methods: A prospective observational study was conducted in 240 patients with American Society of Anesthesiologists physical status classification I and II, and single pregnancy parturients undergoing cesarean section. The patients who had body mass index $(\mathrm{BMI})>35$, estimated blood loss $>1000 \mathrm{~mL}$, needed postoperative bed rest, or received postoperative sedation were excluded. Hyperbaric bupivacaine 8-11 mg and morphine 0.2-0.3 mg were used. The patients were evaluated at the fourth hour until they could sit without adverse events or complete the sixth hour. All patients were evaluated for risk factors delaying the time duration to sit. Results: Out of 240 patients, $77.0 \%, 90.9 \%$, and 98.4\% were able to sit at the fourth, fifth, and sixth hour, respectively. The risk factors that delayed time to sit were Bromage scale $>1$ and pain score $>3$ by the univariate analysis, and were Bromage scale $>1$ and pain score $>3$ by the multivariate analysis.

Conclusion: Seventy-seven percent of the patients could sit at the fourth hour, and most patients (98\%) could sit at the sixth hour. The risk factors that delayed the time duration to sit were Bromage score $>1$ and pain score $>3$.
\end{abstract}

Keywords: Ambulation, cesarean section, spinal anesthesia

Spinal anesthesia has been used to provide anesthesia for cesarean section for a long time. For cesarean section, the use of hyperbaric bupivacaine for subarachnoid block was advocated in text books of anesthesia [1, 2]. Post-spinal anesthetic care protocols requiring prolonged recumbancy have been developed to prevent adverse events from residual block such as dizziness, syncope, or falls. However, early ambulation after surgery is still recommended for its benefits in returning the normal function or decreasing risk of deep vein thrombosis [3]. For this reason, time duration to sitting and ambulation has

Correspondence to: Sahatsa Mandee, MD. Department of Anesthesiology, Faculty of Medicine, Siriraj Hospital, Mahidol University, 2 Arun Amarin Rd., Prannok, Bangkok 10700, Thailand.E-mail: sahatsa2@hotmail.com been studied following various types of ambulatory surgery $[4,5]$. For example, Plug et al. [6] recommended return to pinprick sensation of sacral nerve root fourth and fifth, ability to do plantar flexion (S1), and return of proprioceptive function of the big toes as criteria to start ambulation in patients receiving spinal anesthesia.

Sitting is the first step towards ambulation after surgery and preferable position for lactation, holding the baby, and building maternal-child bonding. In Siriraj Hospital, the most common anesthetic of choice for parturients undergoing cesarean section is spinal anesthesia with $0.5 \%$ bupivacaine $8-12 \mathrm{mg}$ and morphine 0.1-0.3 mg. Nursing practice generally allows patients to sit and start ambulation after 6-12 hours. In previous studies [7, 8], parturient receiving this dosage of bupivacaine should be able to sit and 
ambulate after the fourth hour. However, there is no report to indicate the proportion of patients that could sit at the fourth hour after intrathecal administration. The objective of this study is to investigate the appropriate time duration to sit and to encourage patients to start ambulation without adverse events, and to clarify risk factors that delayed the time duration to sit in this group of patients.

\section{Methods}

This study was approved by the Research Ethics Board of the Faculty of Medicine, Siriraj Hospital, Mahidol University. After having the informed consent, a prospective observational study of 240 parturients scheduled for cesarean section under spinal anesthesia was conducted at Siriraj Hospital.

Eligible patients were 18-40 year, single pregnancy, American Society of Anesthesiologists (ASA) physical status classificationI-II and intraoperative blood loss $<1000 \mathrm{~mL}$. The patients were excluded when they had body mass index (BMI) $>35$, required postoperative bed rest, or received a sedative drug in postoperative period. Two hundred forty parturients were recruited.

All patients were advised about the study protocol and informed consent was obtained. After standard perioperative care for paturients receiving cesarean section, spinal anesthesia was performed with $0.5 \%$ Bupivacaine plus morphine. The actual dose was dependent on anesthesiologist preference.

Demographic data, diagnosis, ASA classification, the intrathecal injection time, dosage of bupivacaine and morphine, site of injection, and number of punctures were recorded. Then level of anesthesia was evaluated and the operation was performed. Total intraoperative fluid, estimated blood loss, and supplemented medication were recorded.

The patients were managed with standard postoperative care in the recovery room and sent to wards after reaching the recovery room discharge criteria. The patients were in the supine position until four hours after the intrathecal injection. At the fourth hour after intrathecal injection, the level of sensory and motor blockade was assessed by cold touch sensation and Bromage scale. This scale is defined as follows:

0 : able to straight leg raise (SLR) and both feet and knee,

1: unable to straight leg raise, able to flex knee and feet,
2: unable to straight leg raise or flex knees, able to move feet,

3: unable to move knees and feet.

Motor recovery also was evaluated by assessing motor power of the plantar flexor muscle, which is controlled by S1.

Sympathetic recovery was assessed by assessing orthostatic hypotension. Blood pressure was measured in supine and then patients were asked to move to the upright sitting position by themselves. At four hour, if patients had orthostatic hypotension (by definition of decreasing of systolic blood pressure at least $20 \mathrm{mmHg}$ or decreasing of diastolic blood pressure at least 10 mmHg within three minutes), presyncope, nausea, vomiting, or sitting without stability, the patient would be declared as unable to sit and asked to lie down. The blood pressure would be rechecked and managed by the investigator. If needed, intravenous fluid loading and a vasopressor would be given and the event time would be recorded.

Investigators evaluated the patients every one hour until patients could sit without orthostatic hypotension and adverse events or until the sixth hour. The presumed risk factors for delayed time duration to sit were collected. Those were sympathetic deficit by evidence of orthostatic hypotension, sensory deficit higher than lumbar level, motor deficit by grading Bromage score $>1[6]$, hemodynamic factor by the estimated volume of blood loss $>500 \mathrm{~mL}$, central nervous system disturbance by the evaluated sedation score, and pain score $>3$ by the evaluated pain score with numerical analog scale.

Sample size calculation was based on the ratio of patients who could not sit at the fourth hour from a pilot study in 20 patients undergoing cesarean section with spinal anesthesia. All calculations assumed a twotailed alpha error of $5 \%$ and $80 \%$ power.

\section{Statistical analysis}

The statistical analysis was performed using the SPSS program (SPSS11.5). Demographic variable was analyzed by descriptive statistics. Categorical variables were analyzed by Chi-square, and multivariate analysis was analyzed by Logistical Regression Analysis.

\section{Results}

Spinal anesthesia was achieved in all parturients. None required general anesthesia because of inadequate anesthesia or developed high spinal block. 
The demographic characteristics of 240 patients are shown in Table 1. There were no differences between two groups of patients who could sit at the fourth hour and could not.

Out of 240 patients, 185 (77.1\%), 218 (90.8\%), and $236(98.3 \%)$ could sit without adverse events at the fourth, fifth, and sixth hour, respectively. Only four patients had orthostatic hypotension at the fourth hour (1.4\%), and no patient had an experience of orthostatic hypotension after reaching the fifth hour. No patients required vasopressors or fluid loading. The risk factors associated with delayed time duration to sit without adverse events at the fourth hour by univariate analysis were Bromage scale $>1$ ( $p<0.01, \mathrm{OR}=5.94$, $95 \% \mathrm{CI}=2.71-13.1)$, pain score $>3(\mathrm{p}<0.01, \mathrm{OR}=55$, $95 \% \mathrm{CI}=7.26-500)$. By multivariate analysis, these were Bromage scale $>1$ ( $p<0.01$, OR=5.16, $95 \% \mathrm{CI}=2.14-12.5)$, and pain score $>3$ ( $<<0.01$, OR=55.5, 95\%CI=6.62-500) (see Table 2).

Level of sensory, motor blockage, sedation score, and pain score are presented in Table 2 . There were no differences between the two groups in sensory level, sedative score, or estimated blood loss between two groups.

Table 1. Demographic data.

\begin{tabular}{lccc}
\hline Demographic data & \multicolumn{2}{c}{ Mean \pm SD } & P-value \\
\cline { 2 - 3 } & Can sit in four hours & Cannot sit in four hours & \\
\hline Age & $30.1 \pm 5.6$ & $29.1 \pm 5.3$ & 0.221 \\
Height (cm) & $157.7 \pm 5.2$ & $157.6 \pm 6.7$ & 0.856 \\
Weight (kg) & $67.5 \pm 10.0$ & $67.2 \pm 12.6$ & 0.868 \\
Duration & $1.5 \pm 0.5$ & $1.4 \pm 0.5$ & 0.20 \\
Volume of Bupivacaine & $2.0 \pm 0.9$ & $2.0 \pm 0.1$ & 0.53 \\
BMI (m/kg) & $27.1 \pm 3.8$ & $27.1 \pm 4.9$ & 0.93 \\
\hline
\end{tabular}

Table 2. Factors affecting inability to sit at the fourth hour after spinal anesthesia with $0.5 \%$ hyperbaric bupivacaine plus morphine

\begin{tabular}{|c|c|c|c|c|c|}
\hline \multirow[t]{2}{*}{ Factor } & \multicolumn{2}{|c|}{ Number } & \multicolumn{2}{|c|}{ Crude analysis } & \multirow{2}{*}{$\begin{array}{l}\text { Adjust OR } \\
95 \% \text { CI }\end{array}$} \\
\hline & $\begin{array}{l}\text { Can sit in } \\
\text { four hours }\end{array}$ & $\begin{array}{l}\text { Cannot sit in } \\
\text { four hours }\end{array}$ & P-value & OR (95\%CI) & \\
\hline \multicolumn{6}{|l|}{ Sensory level } \\
\hline Level: $L$ & $133(75.1 \%)$ & $44(24.9 \%)$ & 0.295 & 1.00 & 1.00 \\
\hline Level: $T$ & $52(82.5 \%)$ & $11(17.5 \%)$ & - & $1.56(0.75-3.30)$ & $1.84(0.78-4.34)$ \\
\hline \multicolumn{6}{|l|}{ Motor level } \\
\hline Bromage: 1 & $171(82.3 \%)$ & $37(17.8 \%)$ & 0 & 1.00 & 1.00 \\
\hline Bromage: 2-3 & $14(43.8 \%)$ & $18(56.3 \%)$ & 0 & $5.94(2.71-13.01)$ & $5.16(2.14-12.5)$ \\
\hline \multicolumn{6}{|l|}{ Sedate awake } \\
\hline Score: 0-1 & 178(78.1\%) & $50(21.9 \%)$ & 0.15 & 1.00 & 1.00 \\
\hline Score: 2-3 & $7(58.3 \%)$ & $5(41.7 \%)$ & & $2.54(0.77-8.35)$ & $2.53(0.63-10.10)$ \\
\hline \multicolumn{6}{|l|}{ Pain score } \\
\hline Score: $\leq 3$ & $184(81.4 \%)$ & $42(48.6 \%)$ & 0.00 & 1.00 & 1.00 \\
\hline Score: >3 & $1(7.1 \%)$ & $13(92.9 \%)$ & & $55(7.26-500)$ & $55.5(6.62-500)$ \\
\hline \multicolumn{6}{|l|}{ Blood loss } \\
\hline$\leq 500 \mathrm{~mL}$ & $151(78.6 \%)$ & $41(21.4 \%)$ & 0.25 & 1.00 & 1.00 \\
\hline$>500 \mathrm{~mL}$ & $34(70.8 \%)$ & $14(29.2 \%)$ & & $1.51(0.74-3.08)$ & $1.56(0.70-3.48)$ \\
\hline
\end{tabular}




\section{Discussion}

In this study, 184 (77.1\%) of 240 patients were able to sit at the $4^{\text {th }}$ hour after spinal anesthesia with $0.5 \%$ hyperbaric bupivacaine plus morphine. Almost all patients (98.4\%) were able to sit without adverse symptoms at the $6^{\text {th }}$ hour. The univariate analysis showed that risk factors to delay the time duration to sit were persistent motor block or inadequate pain control.

Spinal anesthesia with bupivacaine 10-12 mg has been recommended in anesthesia textbooks for cesarean section [1, 2]. A troublesome complication of spinal anesthesia is the residual effect of the block on motor, sensory and sympathetic nervous system [9]. If a sympathetic block persists, it would affect the cardiovascular function that lead to orthostatic hypotension, especially during sudden postural change [10].

Post-cesarean section parturients usually prefer a head-up position rather than remaining supine. Early sitting and ambulation would have benefits in promoting return to normal organ functions, holding the baby, lactation, conversing with family members, and decreasing risk of serious complication such as deep vein thrombosis [11].

The present study showed that the time duration to sit without adverse events was longer compared to those in previous studies [4, 5, 8]. Ogun et al. [7] studied parturients receiving intrathecal $0.5 \%$ bupivacaine $15 \mathrm{mg}$ plus morphine and reported the durations of sensory regression to S2 and complete recovery of motor block were $170 \pm 28$ minutes and $220 \pm 32.4$ minutes, respectively. Choi et al. [8] also reported the time that complete motor recovery (Bromage score $=0$ ) from spinal anesthesia for cesarean section with $0.5 \%$ bupivacaine $8-12 \mathrm{mg}$ plus fentanyl was between $131 \pm 31$ and $156 \pm 23$ minutes. However, their demographic data indicated greater height, weight, and BMI. These might be associated with the shorter time duration to ambulation in those studies. It must be mentioned that in the study by Choi, fentanyl was used as the adjuvant medication for spinal anesthesia. Fentanyl might have lower incidence of nausea or vomiting than spinal morphine.

To date, most previous studies have reported the time duration to ambulation in terms of complete motor recovery and sensory regression to S1. However, these terms might not relate to the clinical time, because patients still have sympathovagal imbalance and impaired balance function. Ponhold et al. [9] found a weak correlation between block height and sympathovagal disturbance. Although the patients in this study did not show orthostatic hypotension after the fifth hour, $9.2 \%$ of patients still were unable to sit due to other symptoms.

One study showed the presence of a disparity between the time duration to recovery of motor function and that to achieve postural control and balance. According to Imarengiaya et al. [12], the time duration to recovery of functional balance is 90 to 120 minutes longer compared with the time duration to gross motor function, and the functional balance remained impaired long after the motor function recovers. In our study, we also found that the residual motor blockage (Bromage score $>1$ ) was the risk factor delaying time to sit.

In our study, we found that inadequate pain control (PS $>3$ ) was a risk factor delaying time to sit and ambulate $(\mathrm{p}<0.01)$. Adequate pain control is required for early ambulation and return to normal function. On the other hand, we also found that sensory level, sedation score, and estimated blood loss were not risk factors delaying time to sit.

A limitation in this study was that intraoperative blood loss was estimated by the anesthesia staff in the operating room, not measured, so it may have been subject to biases. Four patients in this study couldnot sit even in six hours. Two had nausea and vomiting and two had nausea (one patient had a prior history of motion sickness). All of them were treated with antiemetic medication and were able to ambulate the day after surgery.

In conclusion, the time to sit without adverse events in parturients receiving spinal anesthesia with $0.5 \%$ bupivacaine plus morphine was four hours after intrathecal administration for the majority of patients. It is reasonable to encourage sitting at that time. Adequate pain control should be advocated to expedite sitting and ambulation time. Almost all patients should be sitting by six hours. Recently, it was suggested that a lower dose of spinal Bupivacaine (4.5 mg) might be enough for cesarean section [13]. Future research is warranted to determine whether this lower dose may result in earlier sitting and ambulation [13, 14].

\section{References}

1. Kuczkowski KM, Reisner LS. Anesthesia for cesarean section. In Chestnutt DH, editor. Obstetric anesthesia: principles and practice. Philadelphia:Elsevier Mosby, 2004. p. 421-46. 
2. Santos AC, Breveman FR, Finster M. Obstetric anesthesia. In Barash PG, Cullen BF, Stoeling RK editors. Clinical anesthesia. Philadelphia:Lippincott Williams\&Wilkins, 2002. p. 1152-80.

3. Kehlet H. Multimodal approach to control postoperative pathophysiology and rehabilitation. Br J Anaesth. 1997; 78:606-17.

4. Wong J, MarshallS, Chung F, Sinclair D, Song D, Tong D. Spinal anesthesia improves the early recovery profile of patients undergoing ambulatory knee arthroscopy. Can J Anesth. 2001; 48:369-74.

5. Gupta A, Axelsson K, Thorn SE, Matthiessen P, Larsson LG, Holmstorm B, Wattwil M. Low-dose bupivacaine plus fentanyl for spinal anesthesia during ambulatory inguinal herniorrhaphy: a comparison between $6 \mathrm{mg}$ and $7.5 \mathrm{mg}$ of bupivacaine. Acta Anaesthesiol Scand. 2003; 47:13-9.

6. Pflug AE, Aasheim GM, Foster C. Sequence of return of neurological function and criteria for safe ambulation following subarachnoid block (spinal-anaesthetic). Can Anaesth Soc J. 1978; 25:133-9.

7. Ogun CO, Kirgiz EN, Duman A, Okesli S, Akyurek C. Comparison of intrathaecal isobaric bupivacainemorphine and ropivacaine-morphine for caesarean delivery. Br J Anaesth. 2003; 90:659-64.
8. Choi DH, Ahn HJ, Kim MH. Bupivacaine-sparing effect of fentanyl in spinal anaesthesia for cesarean delivery. Reg Anesth Pain Med. 2000; 25:240-5.

9. Paul FW, Alejandro RF. Ambulatory (outpatient) Anesthesia. In Miller RD, editor. Miller’s Anesthesia, $6^{\text {th }}$ ed. Philadelphia:Churchill Livingstone, 2005. p. 2589-94.

10. Bandi E, Weeks S, Carli F. Spinal block levels and cardiovascular changes during post-cesarean section transport. Can J Anesth. 1999; 46:736-40.

11. Panhold HV, Vicenzi MN. Incidence of bradycardia during recovery from spinal anaesthesia: influence of patient position. Br J Anaesth. 1998; 81:723-6.

12. Imarengiaye CO, Song D. Funtional balance is impaired after clinical recovery. Anesthesiology. 2003; 98:511-5.

13. Bryson GL, Macneil R, Jeyaraj LM, Rosaeg OP. Small dose spinal bupivacaine for cesarean delivery does not reduce hypotension but accelerates motor recovery. Can J Anesth. 2007; 54:531-7.

14. Chumnarnkitkosol P, Sirinan C, Samranjai P, Keawjareon M, Sornnil A. Low dose intrathecally bupivacaine for cesarean section. Thai Anesthesiol. 2002; 28:192-7. 